\title{
Wnt4 signaling is associated with the decrease of proliferation and increase of apoptosis during age-related thymic involution
}

\author{
TIANLI WEI, NANNAN ZHANG, ZHIBIN GUO, FENG CHI, YAN SONG and XIKE ZHU \\ Research Center, Shengjing Hospital, China Medical University, Shenyang, Liaoning 110004, P.R. China
}

Received October 30, 2014; Accepted September 1, 2015

DOI: $10.3892 / \mathrm{mmr} .2015 .4343$

\begin{abstract}
The thymus is a central lymphoid organ that is responsible for T-lymphocyte development and maturation. Through negative and positive selection, lymphoid progenitor cells, which initiate from the bone marrow, develop into mature $\mathrm{T}$ cells in the thymus, and are subsequently involved in peripheral cell immunity. It has been reported that the Wnt signaling pathway exists widely in thymic epithelial cells and T lymphocytes. Wnt signaling affects the shape and function of thymic epithelial cells and has an important role in maintaining pro-T-cells, and in the subsequent T-cell differentiation. Previous studies have demonstrated that the Wnt signaling pathway participates in age-associated thymic involution. In the present study alterations in proliferation and apoptosis were investigated in murine thymic cells during aging. The results of the present study demonstrated that the aged thymus was characterized by markedly decreased cell numbers, as well as decreased proliferation and increased apoptosis. Concurrently, age-associated changes in thymic cell number and function were accompanied by a decrease in the transcription levels of Wnt4, and downregulation of forkhead box N1 and B-cell lymphoma-extra large, which are two target genes of the Wnt4 signaling pathway. In vitro studies demonstrated that activation of the Wnt4 signaling pathway promotes mouse thymus epithelial cell 1 (MTEC1) cell proliferation,
\end{abstract}

Correspondence to: Professor Xike Zhu, Research Center, Shengjing Hospital, China Medical University, 36 Sanhao Street, Shenyang, Liaoning 110004, P.R. China

E-mail: zhuxk@sj-hospital.org

Abbreviations: TSCs, thymic stromal cells; TECs, thymic epithelial cells; cTECs, cortical thymic epithelial cells; mTECs, medullary thymic epithelial cells; DN, double negative; DP, double positive; GSK-3, glycogen synthase kinase 3; LEF, lymphoid enhancer factor; TCF, T-cell factors; Dex, dexamethasone; MACS, magnetic cell sorting; PCR, polymerase chain reaction; PI, propidium iodide; CT, threshold cycles; MTEC1, mouse thymus epithelial cell 1; SD, standard deviation; ETP, early thymocyte progenitors; $\mathrm{LiCl}$, lithium chloride

Key words: age-associated thymus involution, thymic epithelial cells, thymocytes, Wnt4, cell proliferation, cell apoptosis and that Wnt4 signaling modulation alleviates dexamethasone-mediated MTEC1 cell apoptosis. These results suggest that normal expression levels of Wnt4 have a critical role in maintaining the balance between cell proliferation and apoptosis. Alterations in the Wnt signaling pathway may disrupt the epithelial network structure of the thymus, eventually leading to microenvironmental damage. Therefore, further studies regarding the effects of the Wnt signaling pathway on thymus development and age-related thymic involution, may be beneficial for improving the health conditions of the elderly.

\section{Introduction}

The thymus is essential for a properly functioning immune response. The three-dimensional structure of the thymic meshwork comprises the cortex and medulla, which are predominantly composed of distinct developing T-cell subsets and thymic stromal cells (TSCs). TSCs are a diverse group of cells that includes cortical thymic epithelial cells (cTECs), medullary (m)TECs, mesenchymal cells, fibroblasts, pericytes, endothelial cells, and adipocytes (1). TECs provide essential microenvironments for the maturation of $\mathrm{T}$ cells. In addition, cTECs are responsible for the attraction of T-cell precursors, commitment of cells to the T-cell lineage, expansion of immature double-negative (DN) thymocytes, and positive selection of double-positive (DP) thymocytes (2). mTECs consist of a heterogeneous population of epithelial cells, which generate a suitable microenvironment for recently positively selected CD4 and CD8 single-positive thymocytes (3).

It is currently unclear whether thymic involution is initiated early on in life; however, thymic involution has severe negative effects on immune responsiveness with progressive age (4). Age-related thymic involution has been shown to be associated with reduced immune surveillance, increased risk of infection and cancer, vaccination failure, and delayed T-cell reconstitution in patients undergoing hematopoietic stem cell transplantations (5-7). The progressive loss of thymic function results in a decrease in adaptive immunity. The predominant causes of age-related thymic involution include: Reduced numbers of hematopoietic stem cells (HSCs), accompanied with intrinsic defects $(8,9)$; reduced TECs and deterioration of the stromal microenvironment $(10,11)$; and extrinsic circulating factors that affect the aged microenvironment, including alterations in hormones, growth factors and cytokines (12). Microenvironmental alterations in the aged thymus 
are considered to be the determining cause for defective T-cell development $(13,14)$. During aging of the immune system, the gradual reduction in the number of naïve T cells is associated with a marked destruction of the epithelial network, including reduced TECs, increased fibroblasts, disrupted thymic perivascular space, and the increasing presence of adipocytes $(10,15,16)$. Thymocytes are the major components of the thymic microenvironment in young individuals; however, adipocytes constitute the bulk of the aged thymic cellular space $(15,16)$. As age increases, the thymus undergoes marked fibrotic and fatty alterations, which result in its transformation into adipose tissue.

Despite studies regarding thymic senescence, the molecular mechanism underlying thymic ageing remains to be elucidated. The formation of an appropriate thymic microenvironment is dependent on interactions between developing thymocytes and TECs, which is known as thymic crosstalk $(17,18)$. Understanding the signaling mechanisms that regulate tissue development and maintenance of TECs may result in the identification of the process of thymic involution. Previous studies have demonstrated that maintenance and functional integrity of the thymic stroma requires stimulation via the Notch, bone morphogenetic protein, and Wnt signaling pathways (19-21). Numerous studies have reported the importance of Wnt signaling and Wnt proteins in the maintenance of various stem cell lineages. For example, Wnt3A deficiency has been shown to result in decreased numbers of HSCs in fetal liver, and decreased self-renewal capacity (22). In addition, the Wnt signaling pathway has been implicated in lineage-commitment and cell-fate regulation during development and aging $(23,24)$. The majority of the members of the Wnt glycoprotein family, which currently consists of 19 molecules, have been implicated in the development of the embryonic thymus, and in the maintenance of adult thymic epithelium (25).

Wnt protein expression has previously been detected in thymocytes and TECs, and their receptors and associated regulatory molecules. In addition, TEC cell lines and primary TECs are both capable of responding to Wnt proteins in vitro (21,25-27). Canonical and non-canonical signaling pathways have been identified, which are initiated by various combinations of the Wnt/Frizzled complex. The most studied signaling pathway is the canonical Wnt pathway, which results in stabilization of $\beta$-catenin via inactivation of the 'destruction complex', which consists of adenomatous polyposis coli, axin, and glycogen synthase kinase 3 (GSK-3) (28). In the absence of Wnt signaling, GSK-3 phosphorylates axin, which culminates in the degradation of $\beta$-catenin (28). In the presence of Wnt signaling, GSK-3 activity is inhibited, resulting in the hypophosphorylation of axin, which protects $\beta$-catenin from degradation and allows it to accumulate (29). Stabilized $\beta$-catenin may then translocate to the nucleus where it targets the lymphoid enhancer factor (LEF)-1, as well as T-cell factors (TCF)-1, TCF-3 and TCF-4. The binding of $\beta$-catenin to LEF or TCF initiates the transcription of numerous genes, including forkhead box (FoxN1), B-cell lymphoma-extra large (Bcl-xL), axin, cyclin D1 and c-Myc (30). The thymic degeneration observed in response to transgenic Dickkopf Wnt signaling pathway inhibitor 1 expression is caused by a loss of TEC stem/progenitor cell maintenance or proliferation of an immature TEC subset (3). In addition, loss of Wnt signaling within
TECs results in a decrease in the K5K8DP TEC subset, which is localized at the cortico-medullary junction, and a reduction in the number of cycling TECs primarily within immature subsets (3). Downregulation of Wnt4 has previously been identified as a trigger for epithelial-mesenchymal transition and pre-adipocyte-differentiation during thymic involution (31).

The aim of the present study was to evaluate the role of the specific Wnt protein, Wnt4, in thymic involution. Wnt4 is one of the most abundant Wnt molecules present in the thymus, and it is produced by both thymocytes and TSCs (25). Previous studies have demonstrated that Wnt4 is responsible for the direct upregulation of FoxN1, which is associated with the differentiation of TECs and the subsequent maintenance of thymic epithelial identity (25). FoxN1 is an essential transcription factor that is required for adequate epithelial morphogenesis, and the capacity of TECs to attract lymphoid precursors from the bone marrow (32). Apoptosis inhibiting factor Bcl-xL is highly expressed in normal mouse thymocytes, whereas in Tcf-1 knockout mice Wnt4 signaling is suppressed, which leads to decreased expression of Bcl-xL and increased apoptosis in thymocytes (33). Exposure to glucocorticoids, similar to physiological aging, results in significant thymic senescence (34). Dexamethasone (Dex) treatment has been shown to trigger Wnt4 and FoxN1 downregulation, leading to the increased expression of preadipocyte-type differentiation markers lamina-associated polypeptide 2 and peroxisome proliferator-activated receptor $\gamma(31,34)$. Furthermore, in a Wnt4-overexpressing TEP1 cell line (35), Wnt4 overexpression effectively suppressed Dex-induced increases in adipocyte markers, and protected thymic epithelium-derived cells against Dex-induced senescence at the molecular level (36). Considering the vital role Wnt4 has in Dex-induced senescence, it was hypothesized that Wnt4 may regulate proliferation and apoptosis within the thymus, and contribute to age-related thymic involution. Using in vitro and in vivo approaches, the present study provided evidence that a decreased expression of Wnt4 in the aging thymus may be one of the molecular triggers underlying the process of age-related thymic senescence.

\section{Materials and methods}

Murine model. C57BL/6 mice were purchased from Charles River Laboratories, Inc. (Wilmington, MA, USA). The mice were 1 month old at the time of purchase, and were housed in the Laboratory Center Animal Ministry of Shengjing Hospital (China Medical University, Shenyang, China) until they had reached 5, 10, 15, or 20 months old. All of the mice were maintained in a specific, pathogen-free environment. There were six mice in each group, (male:female, 1:1) and they were maintained at $21 \sim 27^{\circ} \mathrm{C}$ with $40 \sim 70 \%$ humidity and a $12 / 12 \mathrm{~h}$ light/dark cycle. Animal procedures were performed according to the protocol approved by the Experiment Animal Center of China Medical University, in accordance with the recommendations in the Guide for the Care and Use of Laboratory Animals of the National Institutes of Health (37).

Separation of thymocytes and TSCs. The mice were sacrificed with ether gas anaesthesia, then the pleura was cut open using eye scissors, the thymus was exposed and then it was removed with curved eye tweezers. Thymic lobes were rinsed 
in sterilized phosphate-buffered saline (PBS) a number of times. The lobes were enzymatically digested using $1.5 \mathrm{mg} / \mathrm{ml}$ collagenase II (cat. no. 17101; Gibco Life Technologies, Grand Island, NY, USA), $1 \mathrm{mg} / \mathrm{ml}$ trypsin (cat. no. 25200-056; Gibco Life Technologies) and $0.2 \mathrm{mg} / \mathrm{ml}$ DNase I (cat. no. D5025; Sigma-Aldrich, St. Louis, MO, USA), and were incubated at $37^{\circ} \mathrm{C}$ for $30 \mathrm{~min}$ with intermittent agitation. The resulting single cell suspension was resuspended in buffer (2 mM EDTA in PBS with $0.5 \%$ bovine serum albumin; Gibco Life Technologies) and passed through a $100 \mu \mathrm{m}$ strainer (cat. no. 130-041-407; Miltenyi Biotec $\mathrm{GmbH}$, Bergisch Gladbach, Germany) to remove any remaining undigested tissue. The cells were then purified using magnetic cell sorting (MACS) LS separation columns (Miltenyi Biotec $\mathrm{GmbH}$ ). Cell suspensions were labeled with anti-CD45-fluorescein isothiocyanate (FITC) and washed with MACS buffer (Miltenyi Biotec $\mathrm{GmbH}$ ), followed by incubation with anti-FITC microbeads (Miltenyi Biotec $\mathrm{GmbH}$ ). Thymocytes (positively separated) and TSCs (negatively separated) were subsequently used for total RNA isolation, reverse transcription-quantitative polymerase chain reaction (RT-qPCR) analysis, and flow cytometric analysis.

Proliferation analysis. The mice were intraperitoneally injected with $10 \mathrm{mg} / \mathrm{kg}$ 5-ethynyl-2'-deoxyuridine (EdU; Invitrogen Life Technologies) in PBS. EdU is a nucleoside analog to thymidine, which is incorporated into DNA during active DNA synthesis. Detection is based on a click reaction, also known as a copper catalyzed covalent reaction, between an azide and an alkyne. After $24 \mathrm{~h}$, the thymi of the mice were harvested and single-cell suspensions were purified using MACS, as mentioned above. Thymocytes were stained with CD4-allophycocyanin (APC) (cat. no. 100515; BioLegend, San Diego, CA, USA) and CD8-PerCP (cat. no. 100731; BioLegend) antibodies, whereas TSCs were labeled with EpCAM1-APC (clone G8.8) antibodies (cat. no. 118213; BioLegend). The cells were then prepared for EdU detection, according to the manufacturer's instructions (cat. no. C10425; Invitrogen Life Technologies, Carlsbad, CA, USA). The proliferation and apoptosis of the cells was analyzed using a flow cytometer (FACSCalibur; BD Biosciences, Franklin Lakes, NJ, USA).

Apoptosis analysis. As described above, thymocytes and TSCs were separated using MACS. TSCs were labeled with EpCAM1-APC, whereas thymocytes were stained with CD4-APC and CD8-PerCP. For the early thymocyte progenitors (ETP), freshly isolated thymocytes were stained with phycoerythrin (PE)-conjugated lineage markers, as well as CD25-PE (cat. no. 101903) and CD127-PE (cat. no. 135009), CD117-APC (cat. no. 105811) and CD44-FITC (cat. no. 103021) (BioLegend). For the Annexin V apoptosis assay, the cells were stained with Annexin V-FITC (cat. no. KGA107; Nanjing KeyGen Biotech Co., Ltd., Nanjing, China). Propidium iodide was used to exclude dead cells from the apoptosis assays.

RT-qPCR analysis. Total RNA was extracted from the separated thymocytes and TSCs using TRIzol ${ }^{\circledR}$ reagent (Invitrogen Life Technologies), and reverse transcribed using PrimeScript RT reagent kit (cat. no. RR047A; Takara Bio Inc., Otsu, Japan). For qPCR analysis, ABI 7500 system (Applied Biosystems Life Technologies, Foster City, CA,
USA) and SYBR ${ }^{\circledR}$ Premix Ex Taq ${ }^{T M}$ (cat. no. RR820A; Takara Bio Inc.) were used, with $2 \mu \mathrm{l}$ cDNA. The threshold cycles (CT) for three replicate reactions were determined using Sequence Detection System software (ABI 7500 software, version 2.3; Applied Biosystems Life Technologies), and relative transcription abundance was calculated following normalization with $\beta$-actin. The primer pairs used were: FoxN1, forward 5'-TTCCTCAAGGGCAACCAC-3', reverse 5'-CCCATGTCCACAGGGATC-3'; Wnt4, forward 5'-CTCAAAGGCCTGATCCAGAG-3', reverse 5'-TCACAG CCACACTTCTCCAG-3'; Bcl-xL, forward 5'-GCGTGGAAA GCGTAGACAAGGAGATG-3', reverse 5'-ACTGAAGAG TGAGCCCAGCAGAACC-3'; and $\beta$-actin, forward 5'-GCT GTCCCTGTATGCCTCT-3', and reverse 5'-ATGTCACGC ACGATTTCC-3' (Takara Bio Inc.). The cycling conditions were as follows: Initial denaturation at $95^{\circ} \mathrm{C}$ for $30 \mathrm{sec}$, 45 cycles of $95^{\circ} \mathrm{C}$ for $5 \mathrm{sec}$ and $60^{\circ} \mathrm{C}$ for $20 \mathrm{sec}$.

MTECl cell stimulation. The mouse thymus epithelial cell 1 (MTEC1) cell line originated from a mouse thymic medulla cell line established by the Department of Immunology, Peking University Health Science Center (China). The cells were cultured in medium (Gibco Life Technologies) supplemented with $10 \%$ fetal bovine serum (Gibco Life Technologies) and plated in 24-well cultures to ensure that total cell density $\left(5 \times 10^{6}\right)$ was uniform between the compared samples. The cell lines were separately treated with $30 \mathrm{mM} \mathrm{LiCl}$ (cat. no. 213233; Sigma-Aldrich), $10^{-5} \mathrm{M}$ Dex (cat. no. D4902; Sigma-Aldrich) or $500 \mathrm{ng} / \mathrm{ml}$ mouse recombinant Wnt4 protein (cat. no. 475-WN; R\&D Systems, Inc., Minneapolis, MN USA). For some experiments, the cells were co-cultured in $10^{-5} \mathrm{M}$ Dex and $500 \mathrm{ng} / \mathrm{ml}$ recombinant Wnt 4 protein. The control groups of all of the experiments were cultured in common cell medium. After $24 \mathrm{~h}$, the cells underwent total RNA isolation and flow cytometry.

MTECl cell investigation. Following the treatments previously described, the MTEC1 cell line was investigated. For proliferation analysis, EdU was added to the cell culture medium at a final concentration of $50 \mu \mathrm{M}$, and incubated for $2 \mathrm{~h}$ at $37^{\circ} \mathrm{C}$. The cells were then harvested and EdU detection was conducted according to the manufacturer's instructions (cat. no. C10425; Invitrogen Life Technologies). For apoptosis analysis, MTEC1 cells were stained with Annexin V-FITC (cat. no. KGA107; Nanjing KeyGen Biotech Co., Ltd.). Propidium iodide (PI) was used to exclude dead cells from the apoptosis assays. To determine the mRNA expression levels of FoxN1 and Bcl-xL, RT-qPCR was performed as described above.

Statistical analysis. Data are presented as the mean \pm standard deviation. The differences between various experimental groups were compared using a Student's t-test with GraphPad Prism software, version 5.0 (GraphPad Software, Inc., La Jolla, CA, USA). $\mathrm{P}<0.05$ was considered to indicate a statistically significant difference.

\section{Results}

Significant downregulation of Wnt4 signaling is accompanied by age-related thymic involution. Given that the early stages 
A

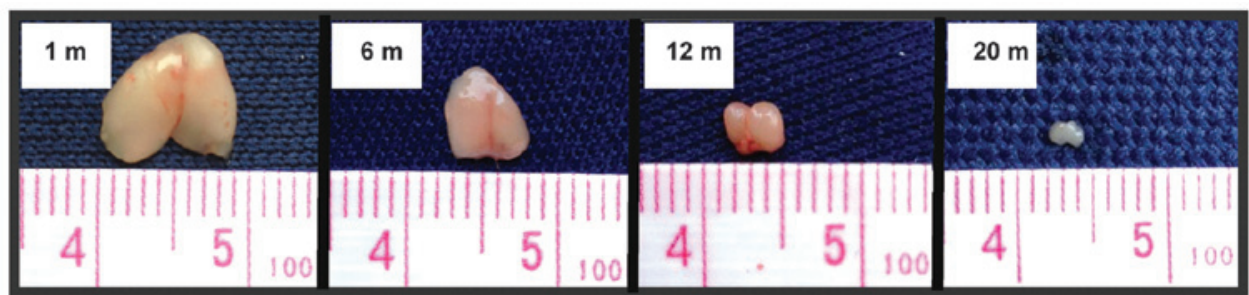

B
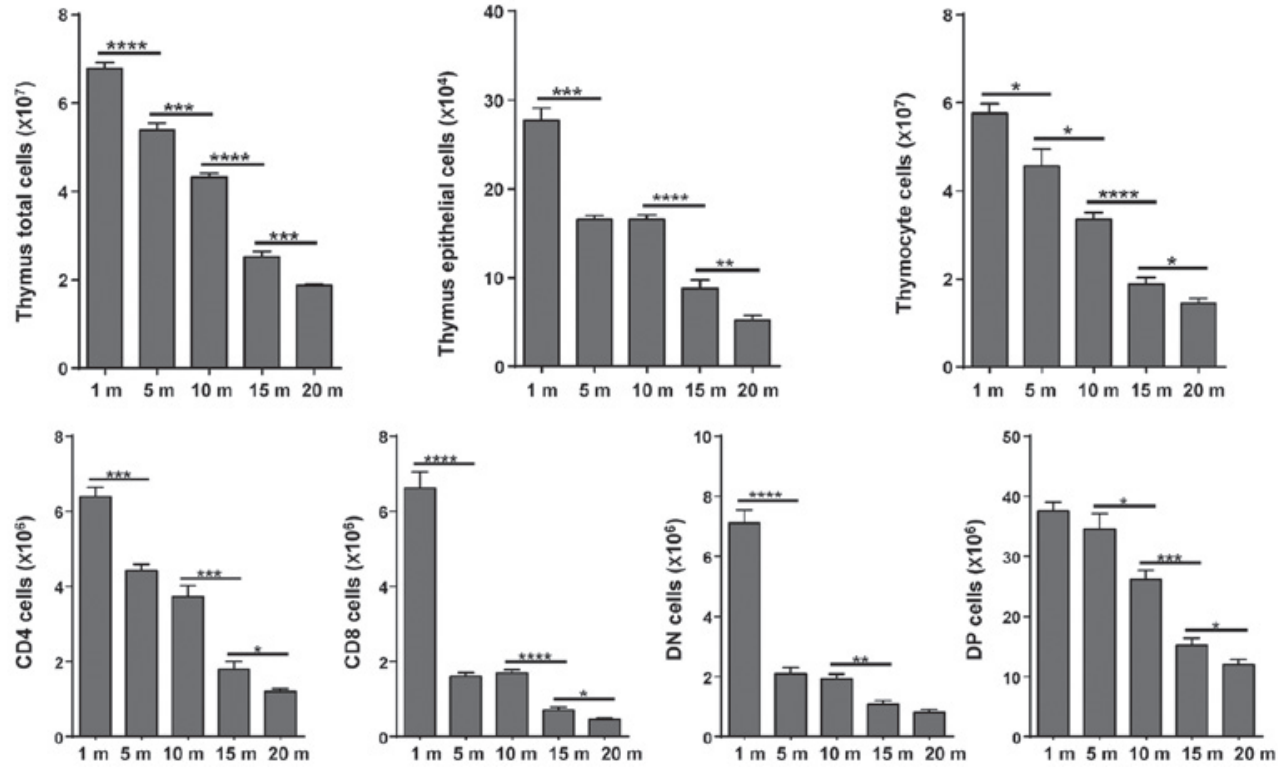

Figure 1. Changes in mouse thymic size and absolute numbers of thymic cells. (A) Comparison of C57BL/6 mouse thymic size at 1, 6, 12 and 20 months. (B) Absolute numbers of thymic total cells, thymic epithelial cells, thymocytes and CD4, CD8, double-negative (DN) and double-positive (DP) cells at 1, 5, 10, 15 and 20 months. Data are presented as the mean \pm standard deviation. Results are representative of six independent experiments $(\mathrm{n}=6) .{ }^{*} \mathrm{P}<0.05 ;{ }^{* *} \mathrm{P}<0.01 ;$ ${ }^{* * * *} \mathrm{P}<0.001 ;{ }^{* * * * *} \mathrm{P}<0.0001$.

A

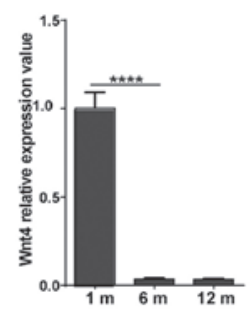

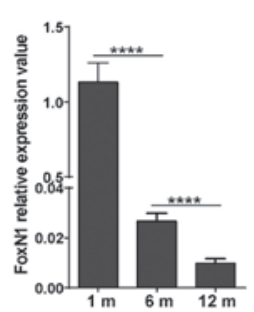

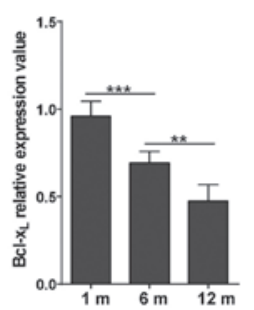

C

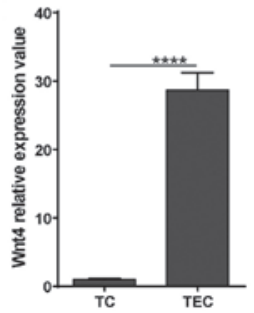

B
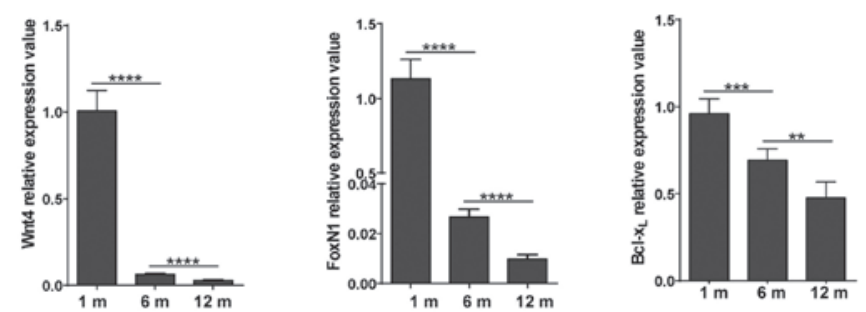

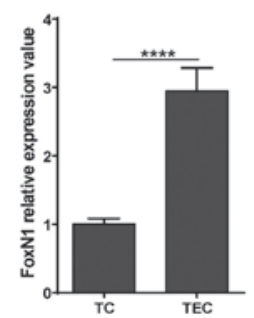

Figure 2. Age-related gene expression alterations in the thymus. Relative mRNA expression levels of Wnt4, forkhead box (Fox)N1 and B-cell lymphoma-extra large (Bcl-xL) in (A) thymic epithelial cells (TEC) and (B) thymocytes (TC) at various ages (1,6 and 12 months), expressed as the mean $2^{-\Delta \Delta C T}$ standardized to the endogenous control $\beta$-actin. (C) Relative mRNA expression levels of Wnt4, FoxN1 compared between the TEC and TC of 1-month old mice. Data are expressed as the mean $2^{-\Delta \Delta C T}$ standardized to the endogenous control $\beta$-actin. Data are presented as the mean \pm standard deviation. Results are representative of six independent experiments $(\mathrm{n}=6)$. ${ }^{* *} \mathrm{P}<0.01 ;{ }^{* * *} \mathrm{P}<0.001 ;{ }^{* * * *} \mathrm{P}<0.0001$.

of T-cell development are highly dependent on the availability of stromal niches (38), and since the number of TECs has been suggested to be the limiting factor in determining thymic size (39), the present study measured the thymic size and the absolute cell numbers of the C57BL/6 mice at various ages (Fig. 1). To detect gene expression alterations, thymocytes 


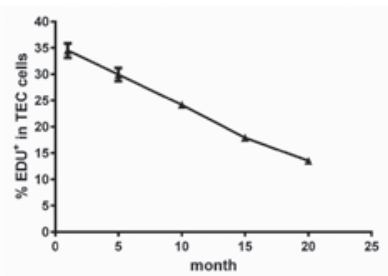

C

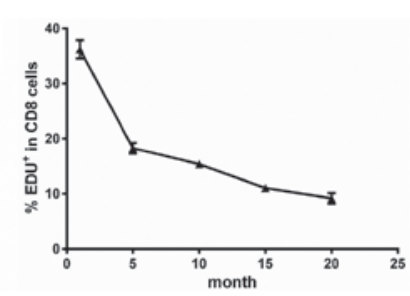

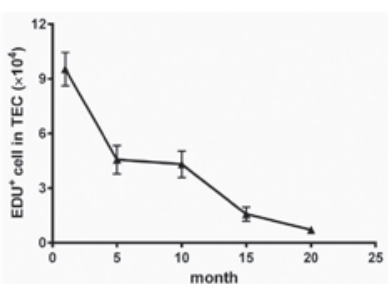

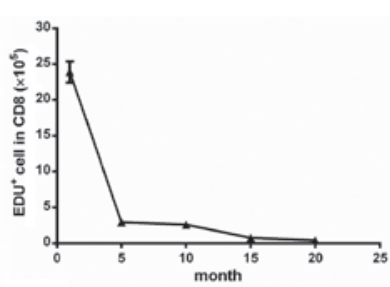

$\mathbf{E}$

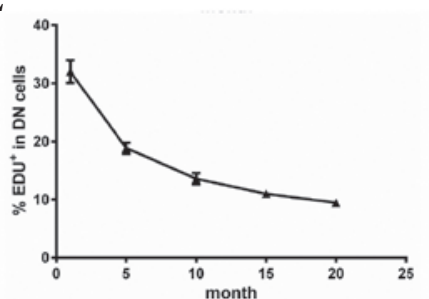

D
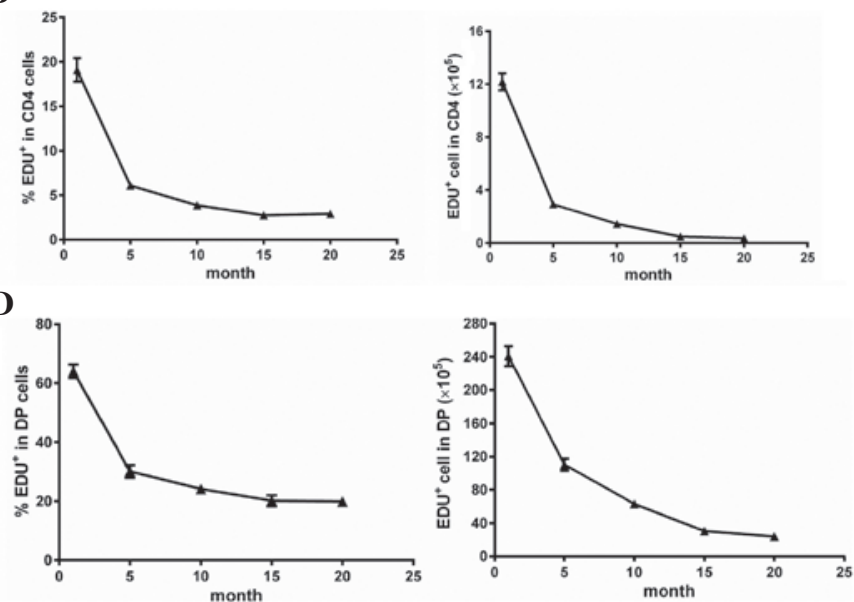

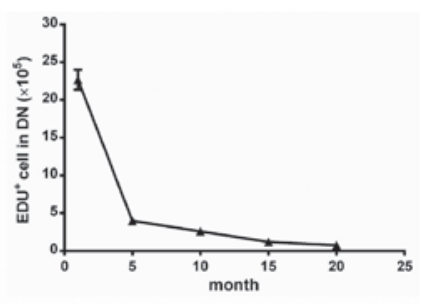

Figure 3. Percentage of proliferation in the thymus at various ages. 5-ethynyl-2'-deoxyuridine (EdU) flow cytometric analysis of proliferation and total number of various thymic cells. (A) Thymic epithelial cells (TECs), (B) CD4, (C) CD8, (D) double-positive (DP), and (E) double-negative (DN) cells. Results are representative of six independent experiments $(n=6)$.

and TECs were purified from 1 month, 6 month and 1-year old C57BL/6 mice based on CD45 expression, and RT-qPCR analysis was performed. Wnt4 signal-related genes, including Wnt4, FoxN1 and Bcl-xL were detected. In addition, Wnt4 and FoxN1 mRNA expression was compared by RT-qPCR between the thymocytes and TECs of 1-month old C57BL/6 mice (Fig. 2).

Thymic size (Fig. 1A) and the absolute numbers of thymocytes and TECs (Fig. 1B) were significantly decreased as the age of the mice increased. Concurrently, the mRNA expression levels of Wnt4, FoxN1 and Bcl-xL decreased in the same manner (Fig. 2A and B). Highly decreased levels of FoxN1 may be caused by the strong downregulation of Wnt4, which occurred by the time the mice reached 1 year of age. Furthermore, Wnt 4 expression was almost 30 times higher in the TECs, as compared with in the thymocytes. Concordantly, the mRNA expression levels of FoxN1 were also more abundant in TECs, as compared with in thymocytes. These results indicate that Wnt4 is predominantly expressed in TECs, and changes in thymic size and absolute numbers of thymic cells are accompanied by a decrease in the transcriptional levels of Wnt4, as well as the expression levels of FoxN1 and Bcl-xL, which are two target genes of the Wnt signaling pathway.

Decreased proliferation and increased apoptosis of thymic cells with age. To analyze the cause of the reduction in thymic cell number, the present study evaluated the proliferation and apoptosis of thymic cells at various ages. TSC composition, as well as organization, is severely disrupted with advancing age. In addition, naïve T-cell production is highest in young individuals and is reduced as thymic involution progresses with age. These processes lead to a significant decrease in the absolute numbers of thymocytes and TECs. With advancing age, the thymus undergoes marked fibrotic and fatty changes, which culminate in its transformation into adipose tissue (40). A balance between cell proliferation and apoptosis can maintain a normal number of cells; therefore, the present study aimed to examine thymic cell proliferation and apoptosis at various ages.

Thymocytes and TSCs were separated from 1-, 5-, 10-, 15and 20-month old C57BL/6 mice, based on CD45 expression. Based on the expression of CD4 and CD8, the proliferation of distinct developing T-cell subsets was determined by EdU-flow cytometric analysis. The proliferation of thymocytes and TECs continuously declined with advancing age (Fig. 3). In particular, the percentage of proliferation was markedly decreased between months 1 and 5, which may provide evidence suggesting that thymic involution is initiated early on in life. Furthermore, Annexin V analysis demonstrated that the apoptosis of TECs and TCs, including CD4, CD8, DP, DN and ETPs, was significantly increased in the aged thymi (Fig. 4).

Wht4 signaling modulation alleviates Dex-induced MTEC1 cell apoptosis. Glucocorticoids are particularly effective immune suppressants, as they induce rapid peripheral $\mathrm{T}$ cell and thymocyte apoptosis, resulting in impaired T cell-dependent immune responses. Primary TECs express glucocorticoid receptors, and high-dosage Dex induces degeneration of the thymic epithelium within $24 \mathrm{~h}$ of treatment (37). The present study aimed to determine how exogenous glucocorticoids affect the thymic epithelial network, and if Wnt4 has an important role in thymic senescence. The MTEC1 cells were treated with $10^{-5} \mathrm{M}$ Dex, or co-cultured in $10^{-5} \mathrm{M}$ Dex and 
A

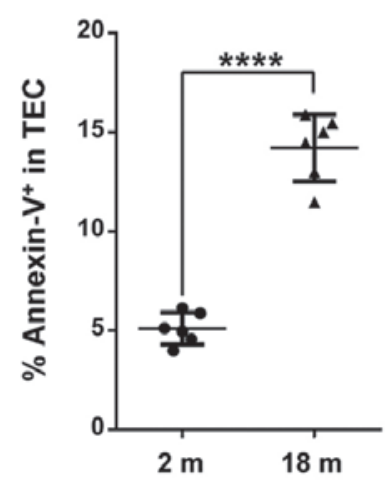

B

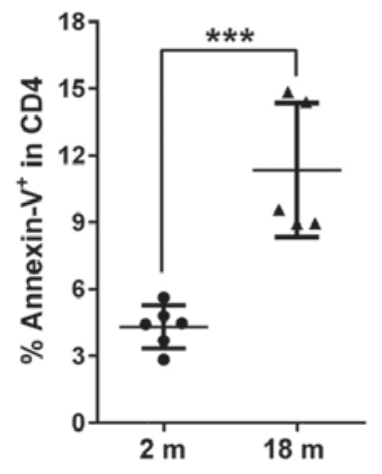

C

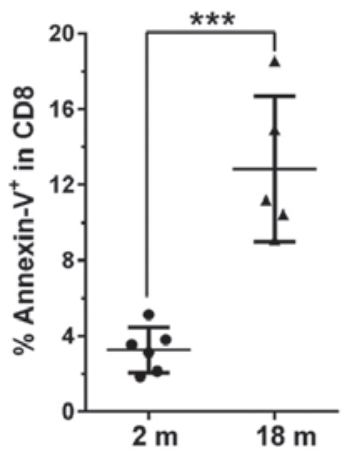

D

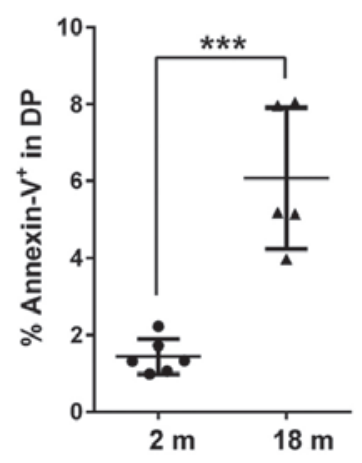

E

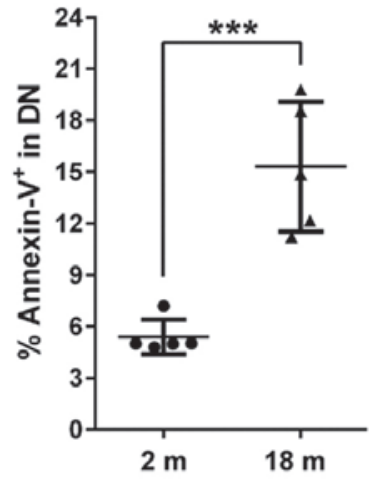

F

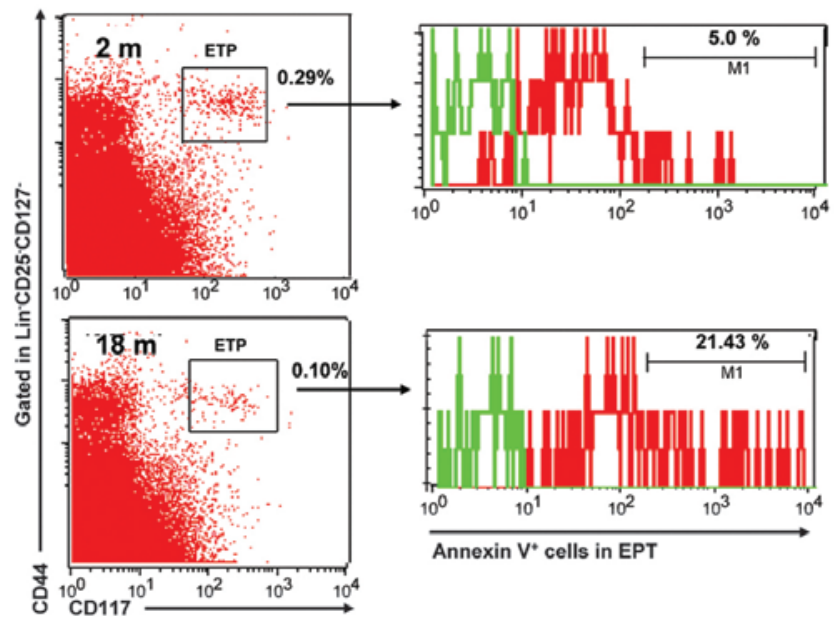

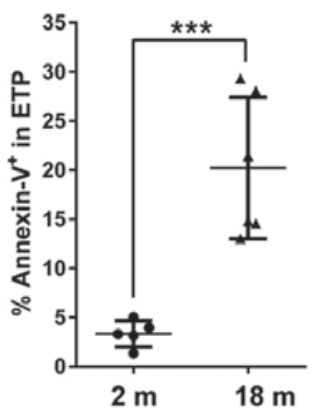

Figure 4. Percentage of apoptotic thymic cells at various ages. Flow cytometric analysis of thymic cells from 2- and 18-month old mice. Apoptotic cells were detected by annexin V and propidium iodide staining (A) Thymic epithelial cells (TECs), (B) CD4, (C) CD8, (D) double-positive (DP), (E) double-negative $(\mathrm{DN})$, and $(\mathrm{F})$ early thymocyte progenitors $(\mathrm{ETP})$. Results are representative of six independent experiments $(\mathrm{n}=6){ }^{* * * *} \mathrm{P}<0.001 ;{ }^{* * * * *} \mathrm{P}<0.0001$.
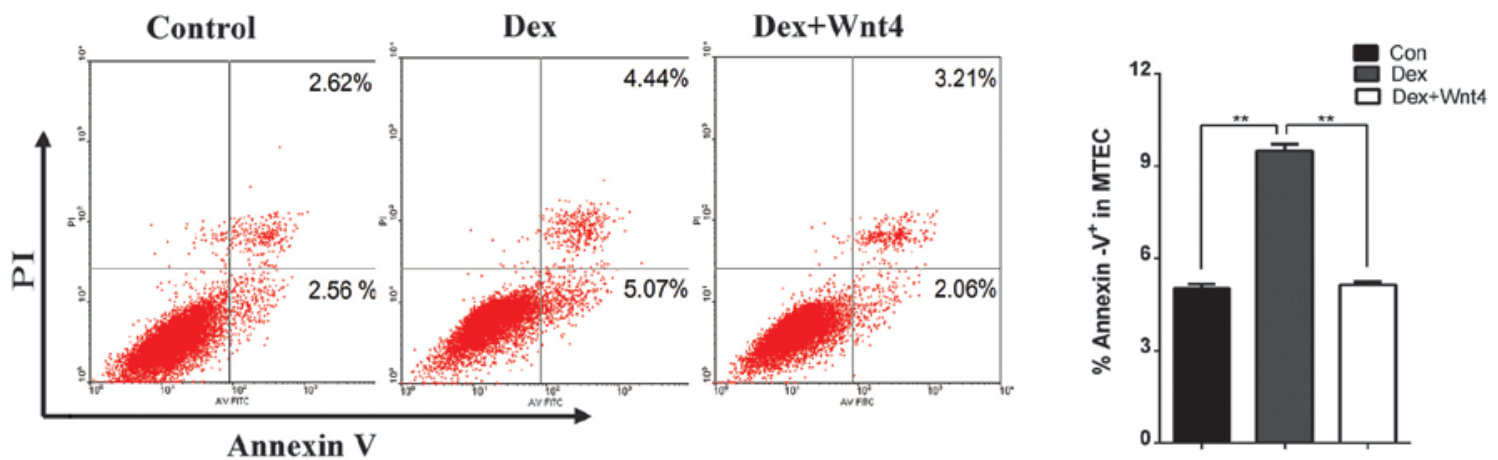

Figure 5. Effects of Wnt4 signaling on dexamethasone (Dex)-mediated mouse thymus epithelial cell 1 (MTEC1) cell apoptosis. The MTEC1 cells were treated with $10^{-5} \mathrm{M}$ Dex, or co-cultured in $10^{-5} \mathrm{M}$ Dex and $500 \mathrm{ng} / \mathrm{ml}$ recombinant Wnt 4 . After $48 \mathrm{~h}$ apoptotic cells were detected by annexin V and propidium iodide (PI) staining. Numbers indicate the percentage of cells in each quadrant. Data are presented as the mean \pm standard deviation. Data shown are representative of at least three independent experiments. ${ }^{* *} \mathrm{P}<0.01$. Con, control.

$500 \mathrm{ng} / \mathrm{ml}$ recombinant Wnt 4 . After $48 \mathrm{~h}$, it was determined whether high glucose altered the rate of apoptosis of MTEC1 cells. As compared with the control group, Dex significantly promoted cell apoptosis, whereas recombinant Wnt4 attenuated Dex-induced cell apoptosis (Fig. 5).

Activation of the Wnt4 signaling pathway promotes MTECl cell proliferation. The biological role of Wnt 4 in
Dex-mediated MTEC1 cell apoptosis was detected in the present study. To determine whether activation of the Wnt4 signaling pathway has a role in cell proliferation, the effects of $\mathrm{LiCl}$ and exogenous Wnt4 were determined. $\mathrm{LiCl}$ is an inhibitor of GSK-3 $\beta$ (41), and thus prevents phosphorylation and degradation of $\beta$-catenin. MTEC1 cells were cultured in medium containing $30 \mathrm{mM} \mathrm{LiCl}$ for $24 \mathrm{~h}$, cell proliferation was then examined using EdU, and the mRNA expression 
A

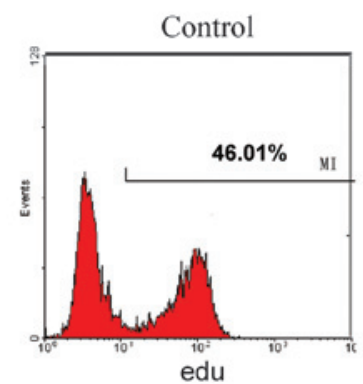

B

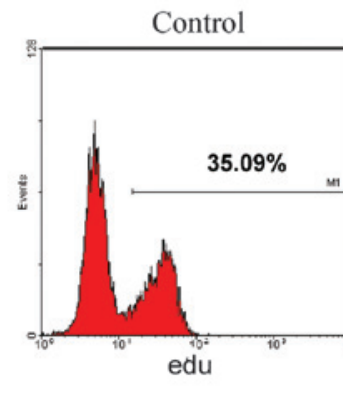

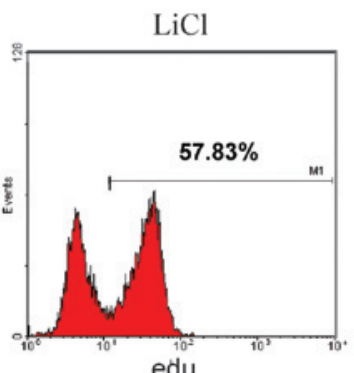

Wnt4

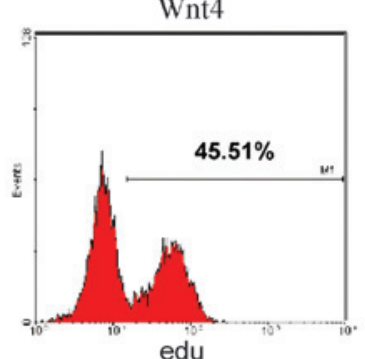

edu
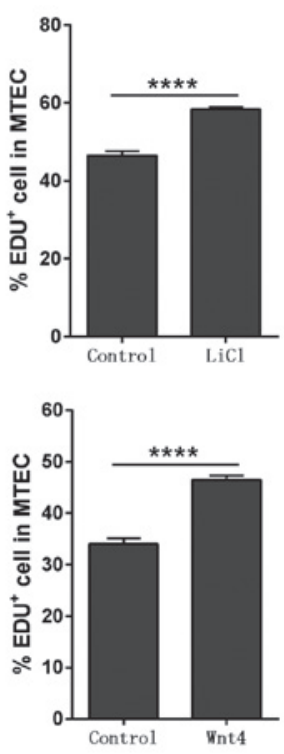

C

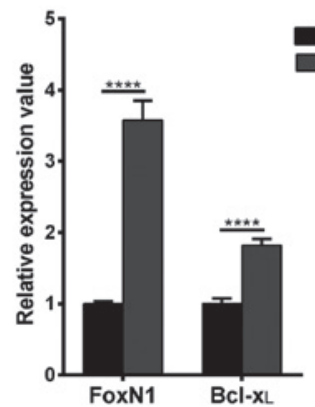

Figure 6. Effects of Wnt4 signaling activation on mouse thymus epithelial cell 1 (MTEC1) cell proliferation. (A) Percentage of cell proliferation after $24 \mathrm{~h}$ incubation in LiCl-supplemented medium, cell proliferation was detected using 5-ethynyl-2'-deoxyuridine (EdU). (B) Percentage of proliferation was quantified following treatment with $500 \mathrm{ng} / \mathrm{ml}$ exogenous Wnt4 protein. (C) Forkhead box (Fox)N1 and B-cell lymphoma-extra large (Bcl-xL) mRNA expression levels were detected using reverse transcription-quantitative polymerase chain reaction following cell culture in LiCl-supplemented medium. Data are presented as the mean \pm standard deviation. Data shown are representative of at least three independent experiments. ${ }^{* * * * *} \mathrm{P}<0.0001$.

levels of Bcl-xL and FoxN1 were determined by RT-qPCR. Concurrently, to determine whether exogenous Wnt4 resulted in increased cell proliferation, MTEC1 cells were examined following incubation with recombinant Wnt4-supplemented media for $16 \mathrm{~h}$. MTEC1 cells cultured in $\mathrm{LiCl}$ and exogenous Wnt4 exhibited $\sim 10 \%$ increased proliferation, as compared with the control group (Fig. 6A and B). As expected, $\mathrm{LiCl}$ treatment alone resulted in an increase in FoxN1 and Bcl-xL mRNA expression levels (Fig. 6C). Notably, cell culture with medium supplemented with $\mathrm{LiCl}$ also resulted in increased proliferation, suggesting that activation of Wnt signaling is a critical step during MTEC1 proliferation, and is not solely limited to the expression of Wnt4.

\section{Discussion}

It has been demonstrated that high-dosage Dex induces degeneration of thymic epithelium within $24 \mathrm{~h}$ of treatment. In addition, the overexpression of Wnt 4 can prevent the upregulation of adipose differentiation-related aging markers, thus suggesting an important role of Wnt 4 in thymic senescence (37). The present study hypothesized that during age-associated thymic involution Wnt4 signaling is associated with thymic proliferation and apoptosis. The results of the present study suggested that enhancing the canonical Wnt4 signal transduction pathway alleviates Dex-mediated MTEC1 cell apoptosis and promotes MTEC1 cell proliferation.

Thymic involution is believed to be initiated early on in life. In the present study, a progressive reduction in thymic size occurred from month 6 , and almost complete thymic involution had occurred by month 20 . TECs provide growth factors and signals that promote the migration and $\mathrm{T}$ lineage commitment of developing lymphocytes. To maintain normal function, the key thymic microenvironment is dependent on proficient cell numbers and normal cell function. By contrast, an imbalance between cell proliferation and apoptosis results in a marked reduction of thymic cell numbers. The present study demonstrated that the aged thymus is characterized by markedly decreased TEC numbers, as well as decreased levels of proliferation, and increased apoptosis of TECs. Notably, the proliferation rate of TECs plummeted from month 5, suggesting that a dysfunctional microenvironment contributes to abnormal thymic function. The lack of a normal thymic microenvironment also results in defective thymic lymphopoiesis in aged thymi. As compared with normal thymic lymphopoiesis in the young thymus, the number of thymocyte sub-populations, especially CD8 and DN, in the elderly mice was markedly reduced after 5 months, alongside decreased 
proliferation and increased apoptosis. These results indicated that T-cell development or proliferation is defective in the aged thymus. With advancing age, normal functional thymocytes and TECs in the thymus are reduced, and adipocytes constitute the bulk of the aged thymic cellular space; the thymus undergoes striking fibrotic and fatty alterations that culminate in its transformation into adipose tissue.

It has been suggested that the absence of Wnt4 may suppress fetal and early postnatal thymic expansion, resulting in decreased TEC numbers, an alteration of the medullary-to-cortical TEC ratio, and a disproportionate loss of immature thymic precursors (42). The present study demonstrated that changes in thymic cell numbers and function are accompanied by a decrease in the transcription levels of Wnt4, as well as the downregulation of FoxN1 and Bcl-xL, which are two target genes of the Wnt signaling pathway. In addition, Wnt4 and FoxN1 were shown to be more abundant in TECs, as compared with thymocytes. A marked decrease of FoxN1 mRNA expression levels was detected in the aged thymus. FOXN1 is a key transcription factor that is responsible for the differentiation of TECs, and its decreased expression may be one reason underlying the reduced proliferation of TECs in elderly mice. Stabilization of $\beta$-catenin, which is a critical coactivator of TCF, enhances DP thymocyte survival via the upregulation of Bcl-xL. Spontaneous or glucocorticoid-induced thymocyte apoptosis has previously been shown to be associated with reduced levels of $\beta$-catenin and Bcl-xL (43). The results of the present study suggested that downregulation of Bcl-xL may result in increased apoptosis of thymocytes and TECs in elderly mice. Concurrently, activation of Wnt4 signaling was shown to result in increased FoxN1 and Bcl-xL mRNA expression levels. In order to further study the role of Wnt4 signaling in cell proliferation and apoptosis, in vitro studies were conducted. The results indicated that activation of the Wnt4 signaling pathway may promote MTEC1 cell proliferation, and alleviate Dex-mediated MTEC1 cell apoptosis. These findings suggested that normal expression level of Wnt4 have a critical role in maintaining the balance between cell proliferation and apoptosis.

In conclusion, based on the present study, it may be suggested that continuously decreasing proliferation and increasing apoptosis occurs during age-related thymic involution. In addition, an age-associated downregulation of Wnt4 may be responsible for the disruption between the balance of cell proliferation and apoptosis. Wnt4 and its downstream signaling pathways may therefore represent interesting candidates to improve thymic function in patients with thymic atrophy.

\section{Acknowledgements}

The authors of the present study would like to thank Professor Yu Zhang (Department of Immunology, Peking University Health Science Center) for providing the MTEC1 cells. The present study was supported by the National Natural Scientific Foundation of China (grant nos. 30872715 and 81270430), the Special Research Fund for Doctoral Program of Education Department of China (grant no. 20112104110011) and the Free Research Program Fund of Shengjing Hospital (grant no. 200805) to X.Z.

\section{References}

1. Petrie HT and Zúñiga-Pflücker JC: Zoned out: Functional mapping of stromal signaling microenvironments in the thymus. Annu Rev Immunol 25: 649-679, 2007.

2. Savage PA and Davis MM: A kinetic window constricts the $\mathrm{T}$ cell receptor repertoire in the thymus. Immunity 14: 243-252, 2001.

3. Osada M, Jardine L, Misir R, Andl T, Millar SE and Pezzano M: DKK1 mediated inhibition of Wnt signaling in postnatal mice leads to loss of TEC progenitors and thymic degeneration. PLoS One 5: e9062, 2010.

4. Dixit VD: Adipose-immune interactions during obesity and caloric restriction: Reciprocal mechanisms regulating immunity and health span. J Leukoc Biol 84: 882-892, 2008.

5. McElhaney JE and Effros RB: Immunosenescence: what does it mean to health outcomes in older adults? Curr Opin Immunol 21: 418-424, 2009.

6. Lynch HE, Goldberg GL, Chidgey A, Van den Brink MR, Boyd R and Sempowski GD: Thymic involution and immune reconstitution. Trends Immunol 30: 366-373, 2009.

7. Holland AM and van den Brink MR: Rejuvenation of the aging T cell compartment. Curr Opin Immunol 21: 454-459, 2009.

8. Min H, Montecino-Rodriguez E and Dorshkind K: Reduction in the developmental potential of intrathymic $\mathrm{T}$ cell progenitors with age. J Immunol 173: 245-250, 2004.

9. Zediak VP, Maillard I and Bhandoola A: Multiple prethymic defects underlie age-related loss of $\mathrm{T}$ progenitor competence. Blood 110: 1161-1167, 2007.

10. Gray DH, Seach N, Ueno T, Milton MK, Liston A, Lew AM, Goodnow CC and Boyd RL: Developmental kinetics, turnover, and stimulatory capacity of thymic epithelial cells. Blood 108: 3777-3785, 2006.

11. Chen L, Xiao S and Manley NR: Foxn1 is required to maintain the postnatal thymic microenvironment in a dosage-sensitive manner. Blood 113: 567-574, 2009.

12. Nobori S, Shimizu A, Okumi M, Samelson-Jones E, Griesemer A, Hirakata A, Sachs DH and Yamada K: Thymic rejuvenation and the induction of tolerance by adult thymic grafts. Proc Natl Acad Sci USA 103: 19081-19086, 2006.

13. Gui J, Zhu X, Dohkan J, Cheng L, Barnes PF and Su DM: The aged thymus shows normal recruitment of lymphohematopoietic progenitors but has defects in thymic epithelial cells. Int Immunol 19: 1201-1211, 2007.

14. Zhu X, Gui J, Dohkan J, Cheng L, Barnes PF and Su DM: Lymphohematopoietic progenitors do not have a synchronized defect with age-related thymic involution. Aging Cell 6: 663-672, 2007.

15. Flores KG, Li J, Sempowski GD, Haynes BF and Hale LP: Analysis of the human thymic perivascular space during aging. J Clin Invest 104: 1031-1039, 1999.

16. Yang H, Youm YH, Sun Y, Rim JS, Galbán CJ, Vandanmagsar B and Dixit VD: Axin expression in thymic stromal cells contributes to age-related increase in thymic adiposity and associated with reduced thymopoiesis independently of ghrelin signaling. J Leukoc Biol 85: 928-938, 2009.

17. van Ewijk W, Holländer G, Terhorst C and Wang B: Stepwise development of thymic microenvironments in vivo is regulated by thymocyte subsets. Development 127 : 1583-1591, 2000.

18. van Ewijk W, Wang B, Hollander G, Kawamoto H, Spanopoulou E, Itoi M, Amagai T, Jiang YF, Germeraad WT, Chen WF and Katsura Y: Thymic microenvironments, 3-D versus 2-D? Semin Immunol 11: 57-64, 1999.

19. Bleul C and Boehm T: BMP signaling is required for normal thymus development. J Immunol 175: 5213-5221, 2005.

20. Anderson G, Pongracz J, Parnell S and Jenkinson EJ: Notch ligand-bearing thymic epithelial cells initiate and sustain Notch signaling in thymocytes independently of $\mathrm{T}$ cell receptor signaling. Eur J Immunol 31: 3349-3354, 2001.

21. Osada M, Ito E, Fermin HA, Vasquez-Cintron E, Venkatesh T, Friedel RH and Pezzano M: The Wnt signaling antagonist Kremen1 is required for development of thymic architecture. Clin Dev Immunol 13: 299-319, 2006.

22. Luis TC, Weerkamp F, Naber BA, Baert MR, de Haas EF, Nikolic T, Heuvelmans S, De Krjiger RR, van Dongen JJ and Staal FJ: Wnt3a deficiency irreversibly impairs hematopoietic stem cell self-renewal and leads to defects in progenitor cell differentiation. Blood 113: 546-554, 2009. 
23. Manolagas SC and Almeida M: Gone with the Wnts: Beta-catenin, T-cell factor, forkhead box $\mathrm{O}$, and oxidative stress in age-dependent diseases of bone, lipid, and glucose metabolism. Mol Endocrinol 21: 2605-2614, 2007.

24. Kléber M and Sommer L: Wnt signaling and the regulation of stem cell function. Curr Opin Cell Biol 16: 681-687, 2004.

25. Balciunaite G, Keller MP, Balciunaite E, Piali L, Zuklys S, Mathieu YD, Gill J, Boyd R, Sussman DJ and Holländer GA: Wnt glycoproteins regulate the expression of FoxN1, the gene defective in nude mice. Nat Immunol 3: 1102-1108, 2002.

26. Pongracz J, Hare K, Harman B, Anderson G and Jenkinson EJ: Thymic epithelial cells provide WNT signals to developing thymocytes. Eur J Immunol 33: 1949-1956, 2003.

27. Weerkamp F, Baert MR, Naber BA, Koster EE, de Haas EF, Atkuri KR, van Dongen JJ, Herzenberg LA and Staal FJ: Wnt signaling in the thymus is regulated by differential expression of intracellular signaling molecules. Proc Natl Acad Sci USA 103: 3322-3326, 2006.

28. Logan CY and Nusse R: The Wnt signaling pathway in development and disease. Annu Rev Cell Dev Biol 20: 781-810, 2004

29. Cadigan KM and Liu YI: Wnt signaling: Complexity at the surface. J. Cell Sci 119: 395-402, 2006.

30. Miller JR: The Wnts. Genome Biol 3: REVIEWS3001, 2002.

31. Kvell K, Varecza Z, Bartis D, Hesse S, Parnell S, Anderson G, Jenkinson EJ and Pongracz JE: Wnt4 and LAP2alpha as pacemakers of thymic epithelial senescence. PLoS One 5: e10701,2010.

32. Hirokawa K, Utsuyama M, Kasai M, Kurashima C, Ishijima S and Zeng YX: Understanding the mechanism of the age-change of thymic function to promote $\mathrm{T}$ cell differentiation. Immuno Lett 40: 269-277, 1994.

33. Ioannidis V, Beermann F, Clevers $\mathrm{H}$ and Held $\mathrm{W}$ : The beta-catenin - TCF-1 pathway ensures CD4(+)CD8(+) thymocyte survival. Nat Immunol 2: 691-697, 2001.
34. Youm YH, Yang H, Sun Y, Smith RG, Manley NR, Vandanmagsar B and Dixit VD: Deficient ghrelin receptor-mediated signaling compromises thymic stromal cell microenvironment by accelerating thymic adiposity. J Biol Chem 284: 7068-7077, 2009.

35. Beardsley TR, Pierschbacher M, Wetzel GD and Hays EF: Induction of T-cell maturation by a cloned line of thymic epithelium (TEPI). Proc Natl Acad Sci USA 80: 6005-6009, 1983.

36. Guide for the Care and Use of Laboratory Animals. 8th edition. National Academies Press, Washington, DC, 2011.

37. Talaber G, Kvell K, Varecza Z, Boldizsar F, Parnell SM, Jenkinson EJ, Anderson G, Berki T and Pongracz JE: Wnt-4 protects thymic epithelial cells against dexamethasone-induced senescence. Rejuvenation Res 14: 241-248, 2011.

38. Prockop SE and Petrie HT: Regulation of thymus size by competition for stromal niches among early $\mathrm{T}$ cell progenitors. J Immunol 173: 1604-1611, 2004.

39. Jenkinson WE, Bacon A, White AJ, Anderson G and Jenkinson EJ: An epithelial progenitor pool regulates thymus growth. J Immunol 181: 6101-6108, 2008.

40. Dixit VD: Thymic fatness and approaches to enhance thymopoietic fitness in aging. Curr Opin Immunol 22: 521-528, 2010.

41. Hocevar BA, Mou F, Rennolds JL, Morris SM, Cooper JA and Howe PH: Regulation of the Wnt signaling pathway by disabled-2 (Dab2). EMBO J 22: 3084-3094, 2003.

42. Heinonen KM, Vanegas JR, Brochu S, Shan J, Vainio SJ and Perrault C: Wnt4 regulates thymic cellularity through the expansion of thymic epithelial cells and early thymic progenitors. Blood 118: 5163-5173, 2011

43. Xie H, Huang Z, Sadim MS and Sun Z: Stabilized beta-catenin extends thymocyte survival by up-regulating Bcl-xL. J Immunol 175: 7981-7988, 2005. 